\title{
MAXIMAL COMPACT NORMAL SUBGROUPS
}

\author{
M. R. PEYROVIAN
}

\begin{abstract}
The main concern is the existence of a maximal compact normal subgroup $K$ in a locally compact group $G$, and whether or not $G / K$ is a Lie group. $G$ has a maximal compact subgroup if and only if $G / G_{0}$ has. Maximal compact subgroups of totally disconnected groups are open. If the bounded part of $G$ is compactly generated, then $G$ has a maximal compact normal subgroup $K$ and if $B(G)$ is open, then $G / K$ is Lie. Generalized FCgroups, compactly generated type I IN-groups, and Moore groups share the same properties.
\end{abstract}

Introduction. We shall be concerned with the conditions under which a locally compact group $G$ possesses a maximal compact normal subgroup $K$ and whether or not $G / K$ is a Lie group. It is known that locally compact connected groups and compactly generated Abelian groups have maximal compact normal subgroups with Lie factor groups. Moreover, almost connected groups (i.e., when $G / G_{0}$ is compact, where $G_{0}$ is the identity component of $G$ ) and compactly generated $F C$ groups share these results.

For general locally compact Abelian groups, the corresponding assertion is not true even if the group is $\sigma$-compact. Consider any $\Omega_{a}$ of $a$-adic numbes; $\Omega_{a}$ is a locally compact $\sigma$-compact Abelian group which has no maximal compact (normal) subgroup.

In this paper we show that a locally compact group $G$ has a maximal compact subgroup if and only if $G / G_{0}$ has (Theorem 1 ). This generalizes substantially the corresponding result for almost connected groups mentioned before. It also reduces the question of the existence of maximal compact subgroups to that of totally disconnected groups-in which we show every maximal compact subgroup is open (Corollary 1 of Theorem 2). In [1] it is proved that a generalized $F C$ group $G$ has a maximal compact normal subgroup $K$. Here we show that $G / K$ is a Lie group (Theorem 5). We also prove that, if the bounded part of $G, B(G)$, is compactly generated, then $G$ has a maximal compact normal subgroup (Theorem 6). Particularly, we see that compactly generated Moore groups, type I IN-groups, and compact extensions of compactly generated nilpotent groups have the desired properties (Theorems 7 and 8). Finally some sufficient conditions for $G / K$ to be a Lie group are summarized in Theorem 10.

If a topological group $G$ has a maximal compact subgroup $N$, then clearly $g N g^{-1}$ is a maximal compact subgroup of $G$ for any $g \in G$. A maximal compact subgroup contains every compact normal subgroup. Hence $K=\bigcap_{g \in G} g N g^{-1}$ is the maximal compact normal subgroup of $G$.

Received by the editors July 12, 1985 and, in revised form, December 24, 1985.

1980 Mathematics Subject Classification (1985 Revision). Primary 22D05. 
THEOREM 1. Let $G$ be a locally compact group. Then $G$ has maximal compact subgroups if and only if $G / G_{0}$ has.

Proof. Suppose $G$ has a maximal compact subgroup $K$. The image of $K$ under the natural mapping $\varphi: G \rightarrow G / G_{0}$ is the compact subgroup $K G_{0} / G_{0}$. Suppose there is a compact subgroup $M / G_{0}$, where $M$ is a closed subgroup of $G$ containing $G_{0}$, such that $K G_{0} / G_{0} \subset M / G_{0}$. Since $M_{0}=G_{0}, M / M_{0}$ is compact. Let $N$ be a compact normal subgroup of $M$ such that $M / N$ is a Lie group [5, p. 175]. Then $M / N$ has a finite number of connected components and $K / N$ is a maximal compact subgroup of $M / N$. So $M / N=(K / N)\left(G_{0} N / N\right)$ by [6, Theorem 3.1]. It follows that $M=K G_{0}$, i.e., $K G_{0} / G_{0}$ is maximal in $G / G_{0}$.

Conversely, suppose $G / G_{0}$ has a maximal compact subgroup $M / G_{0}$. Using Theorem 3.1 of $[6]$ and the previous argument, let $K$ be a maximal compact subgroup of $M$ and $M=K G_{0}$. If $K^{\prime}$ is a compact subgroup of $G$ and $K^{\prime} \supsetneqq K$, then $M / G_{0} \varsubsetneqq K^{\prime} G_{0} / G_{0}$. This contradicts the maximality of $M / G_{0}$ in $G / G_{0}$. Therefore $K$ is a maximal compact subgroup of $G$.

The mere assumption that $G / G_{0}$ has a maximal compact subgroup and hence $G$ has a maximal compact normal subgroup $K$ does not imply that $G / K$ is a Lie group [1, Example 2].

Another aspect of Theorem 1 is that, in a sense, now one needs only to determine when a totally disconnected group has a maximal compact subgroup. We show that such a subgroup, if it exists, is open.

THEOREM 2. In a locally compact totally disconnected group $G$, every compact subgroup is contained in a compact open subgroup of $G$.

ProOF. Let $K$ be a compact subgroup of $G$, and let $H$ be an open compact subgroup of $G$. There is a neighborhood $V$ of $e$ such that $k V k^{-1} \subset H$ for all $k \in K$. Choose a compact open subgroup $N \subset V$, and let $M$ be the closure of the subgroup generated by $\bigcup_{k \in K} k N k^{-1}$. Then $M$ is a compact subgroup, $K M$ is a compact open subgroup of $G$, and $K \subset K M$.

COROLLARY 1. In a locally compact totally disconnected group $G$, every maximal compact subgroup is open.

COROLLARY 2. If $G$ is a locally compact group and $K$ is a maximal compact subgroup of $G$, then $K G_{0}$ is open.

PROOF. $K G_{0} / G_{0}$ is a maximal compact subgroup of the totally disconnected group $G / G_{0}$. Since $K G_{0} / G_{0}$ is open in $G / G_{0}, K G_{0}$ is open in $G$.

DEFINITIONS. (1) An element $g \in G$ is called bounded if the conjugacy class $\left\{x g x^{-1}: x \in G\right\}$ has a compact closure. The set of all bounded elements of $G$ is denoted by $B(G)$. A topological group is an $F C$-group if $G=B(G)$.

(2) An element $g \in G$ is called periodic if it is contained in a compact subgroup of $G$. The set of all periodic elements of $G$ is denoted by $P(G)$.

(3) A locally compact group $G$ is a generalized $F C$-group of length $n$ if there exists a finite sequence $A_{1}, A_{2}, \ldots, A_{n+1}$ of closed normal subgroups of $G$ such that $G=A_{1} \supset A_{2} \supset \cdots \supset A_{n+1}=\{e\}$ and each factor $A_{i} / A_{i+1}$ is a compactly generated $F C$-group.

(4) A locally compact group $G$ is pro-Lie if every neighborhood of the identity contains a compact normal subgroup $K$ such that $G / K$ is a Lie group. 
LEMMA 3. Let $G$ be a locally compact group and $N$ a closed normal subgroup of $G$. If $K$ and $K^{\prime}$ are maximal compact normal subgroups of $G$ and $N$ respectively, then $K^{\prime}=K \cap N$. In particular, if $K \subseteq N$, then $K=K^{\prime}$.

ProOF. $K \cap N$ is a compact normal subgroup of $N$. Hence $K \cap N \subseteq K^{\prime}$. It is now sufficient to prove that $K^{\prime}$ is a normal subgroup of $G$. Given any $g \in G$, $g K^{\prime} g^{-1}$ is a compact subgroup of $N$; and for $n \in N$,

$$
n\left(g K^{\prime} g^{-1}\right) n^{-1}=g\left(g^{-1} n g\right) K^{\prime}\left(g^{-1} n g\right)^{-1} g^{-1}=g K^{\prime} g^{-1}
$$

since $g^{-1} n g \in N$. It follows that $g K^{\prime} g^{-1}$ is a compact normal subgroup of $N$. Therefore $g K^{\prime} g^{-1} \subseteq K^{\prime}$ and this shows that $K^{\prime}$ is normal in $G$. The lemma is thus proved.

LEMMA 4. Let $G$ be a locally compact group which has a maximal compact normal subgroup $K$. Let $H$ be a closed normal compactly generated subgroup of $G$ which has a maximal compact normal subgroup $P$ and $H / P$ is a pro-Lie group. If $G / H$ is compact, then $G / K$ is a Lie group.

Proof. By Lemma 3, $P$ is a normal subgroup of $G$. Let $G^{\prime}=G / P, H^{\prime}=H / P$, and $K^{\prime}=K / P$. Now $G / H \cong G^{\prime} / H^{\prime}$, thus $G^{\prime} / H^{\prime}$ is compact, where $H^{\prime}$ is a closed normal compactly generated pro-Lie group. By Theorem 4 of $[\mathbf{3}], G^{\prime}$ is also proLie. But $G^{\prime} / K^{\prime} \cong G / K$, hence $G / K$ is a pro-Lie group with no nontrivial compact normal subgroup. It follows that $G / K$ is a Lie group.

THEOREM 5. A generalized $F C$-group $G$ has a maximal compact normal subgroup $K$ and $G / K$ is a Lie group.

ProOF. The existence of $K$ is proved in [1, Theorem 3]. To prove that the corresponding factor group is Lie, consider the chain $G=A_{1} \supset A_{2} \supset \cdots \supset A_{n} \supset$ $A_{n+1}=\{e\}$ as in the definition. We proceed by induction, noting that, for $n=1$, the conclusion follows from [2, Theorem 3.20]. We assume that every generalized $F C$-group with sequence $\left\{A_{i}\right\}$ of length less than or equal to $n$ has the desired property. Thus $G / A_{n}$ has a maximal compact normal subgroup $N / A_{n}$ such that $\left(G / A_{n}\right) /\left(N / A_{n}\right) \cong G / N$ is a Lie group, where $N$ is a closed normal subgroup of $G$ containing $A_{n}$. As a compactly generated $F C$-group, $A_{n}$ has a maximal compact normal subgroup $P$ and $A_{n} / P$ is a Lie group. Since $P$ is maximal in $A_{n}$, it is closed and normal in $G$. Let $\varphi: G \rightarrow G / A_{n}$ be the natural mapping. Then, $\varphi(K) \subset N / A_{n}$, so $K \subset N$.

Now $N$ is a generalized $F C$-group with sequence $N \supset A_{n} \supset\{e\}$. Thus $N$ has a maximal compact normal subgroup which must be $K$, by Lemma 3 , since $K \subset N$. The groups $N, A_{n}, K$, and $P$ satisfy the conditions of Lemma 4 ; hence $N / K$ is a Lie group. Since $G / N$ is a Lie group and $G / N \cong(G / K) /(N / K), G / K$ is also a Lie group.

COROLLARY 1. Let $G$ be a locally compact group and $H$ a compactly generated normal $F C$-subgroup of $G$. If $G / H$ is compact, then $G$ has a maximal compact normal subgroup $K$ and $G / K$ is a Lie group.

Proof. Observe that $G / H$ is a compact $F C$-group, thus $G$ is a generalized $F C$-group with sequence $G \supset H \supset\{e\}$. 
COROLlARY 2. Let $G$ be a compactly generated group such that $G / \overline{B(G)}$ is compact. Then $G$ has a maximal compact normal subgroup $K$ and $G / K$ is a Lie group.

Proof. Let $H=\overline{B(G)}$. Then $H$ is compactly generated. It follows that $H$ is an $F C$-group by Proposition 4 of [8]. Thus, by Corollary 1 , the conclusion is obtained.

We derive another corollary for later use. A locally compact group $G$ is called a $Z$-group if $G / Z(G)$ is compact, where $Z(G)$ is the center of $G$.

COROLLARY 3. Let $G$ be a locally compact group and $H$ a compactly generated $Z$-subgroup of $G$. If $G / H$ is compact, then $G$ has a maximal compact normal subgroup $K$ and $G / K$ is a Lie group.

ProOF. Immediate from Corollary 1.

The following example shows that the result of Corollary 1 is not covered already.

EXAMPLE. Let $G=\mathbf{R}^{2} \times_{\eta} T$, where $T$ is the unit circle and acts on $\mathbf{R}^{2}$ by rotation, i.e., the automorphism of $\mathbf{R}^{2}$ induced by $e^{i \theta} \in T$ is a rotation of $\mathbf{R}^{2}$ by the angle $\theta$. If $H=\mathbf{R}^{2}$, then $H$ is a compactly generated $F C$-group and $G / H$ is compact. Since $(x, y, 0)(0,0, \pi)(-x,-y, 0)=(2 x, 2 y, \pi)$, the conjugacy class of $(0,0, \pi)$ contains a nonrelatively compact set. Thus $G$ is not an $F C$-group. But $G$ is a Lie group with the identity as the maximal compact normal subgroup.

Let $G$ be locally compact. Then $B(G) \cap P(G)$ is a normal subgroup generated by all compact normal subgroups of $G[\mathbf{7}$, Corollary 5.6]. Hence, if $K$ is the maximal compact normal subgroup of $G$, then $K=P(G) \cap B(G)$.

THEOREM 6. If $G$ is a locally compact group and $B(G)$ is compactly generated, then $G$ has a maximal compact normal subgroup.

Proof. $\overline{B(G)}$ is compactly generated and hence by Proposition 4 of [8], it is an $F C$-group. Thus $\overline{B(G)}$ has a maximal compact normal subgroup $K$. As in the proof of Lemma 3, we can see that $K$ is normal in $G$. Suppose $K$ is not maximal. Then there is a compact normal subgroup $N$ of $G$ such that $K \subset N$ and $K \neq N$. But $N \subset P(G) \cap B(G) \subset \overline{B(G)}$. This contradicts the maximality of $K$ in $\overline{B(G)}$. It follows that $K$ is the maximal compact normal subgroup of $G$.

The converse of this theorem does not hold. In Example 1 of $[\mathbf{1}], B(G)=$ $\sum H_{i} \times\{1\}$ is not compactly generated. This theorem can be applied in abstract group theory:

COROLlary. Let $G$ be a group. If $B(G)$, the set of elements of $G$ which have a finite conjugacy class, is finitely generated, then $G$ has a maximal finite normal subgroup.

If $B(G)$ is compactly generated and open in $G$, then $G$ is an IN-group by Theorem $1[8]$. Thus $G / K$ is a Lie group.

The next several results, which establish sufficient conditions for a group to have a maximal compact normal subgroup, are expressed in terms of representations of a group.

DEFINITIONS. Let $G$ be a locally compact group.

(1) $G$ is called a Moore group if $G$ has only finite dimensional irreducible (continuous unitary) representations. 
(2) $G$ is called a type I group if the von Neumann algebra generated by any representation of $G$ is type I.

THEOREM 7. Let $G$ be a compactly generated type I IN-group. Then $G$ has a maximal compact normal subgroup $K$ and $G / K$ is a Lie group.

PROOF. It is known that a compactly generated group has a compact normal subgroup $N$ such that $G^{\prime}=G / N$ is second countable. Since $G^{\prime} / B\left(G^{\prime}\right)$ is finite by Proposition 4.1 of $[4]$, it follows that $G^{\prime}$ has a maximal compact normal subgroup $M$, by Corollary 2 of Theorem 5 . Then $K=\varphi^{-1}(M)$ is the maximal compact normal subgroup of $G$, where $\varphi$ is the natural mapping of $G$ onto $G^{\prime}$. Now $G / K$ is a Lie group since $G$ is an IN-group.

As a result, every compactly generated type I MAP- or SIN-group shares the conclusion of Theorem 7. The same assertion follows for compactly generated Moore groups, since a Moore group is a type I SIN-group.

THEOREM 8. Let $G$ be a locally compact group. Let $H$ be a compactly generated nilpotent normal subgroup of $G$ such that $G / H$ is compact. Then $G$ has a maximal compact normal subgroup $K$ and $G / K$ is a Lie group.

Proof. Since $G$ is pro-Lie by $[\mathbf{3}$, Theorem 9$]$, we only need to show the existence of $K$. For this purpose, we use induction on the nilpotency class $n$ of $H$. For $n=1$, $H$ is Abelian and the conclusion follows from Corollary 3 of Theorem 5. Suppose the assertion is true for groups of class less than $n$. By [3, Proposition 8], $Z(H)$ is compactly generated. Clearly $H / Z(H)$ is compactly generated with nilpotency class less than $n$. Thus $G / Z(H)$ has a maximal compact normal subgroup $M / Z(H)$, where $M$ is a closed normal subgroup of $G$. Then $G / M \cong(G / Z(H)) /(M / Z(H))$ has no nontrivial compact normal subgroup. Hence it is sufficient to show that $M$ has a maximal compact normal subgroup. But this follows from Corollary 3 of Theorem 5, since $M$ and $Z(H)$ satisfy its hypotheses. Hence the proof is complete.

On many occasions we find the following lemma very useful.

LEMMA 9. Let $G$ be a locally compact group. Suppose

(1) $G$ has a maximal compact normal subgroup $K$,

(2) $N$ is a $\sigma$-compact closed normal subgroup of $G$ and has a maximal compact normal subgroup $K_{1}$ such that $N / K_{1}$ is a Lie group, and

(3) $G / N$ is a Lie group.

Then $G / K$ is a Lie group.

Proof. Observe that $K_{1}=K \cap N$. Since $N$ is $\sigma$-compact, $K N / K \cong N / K \cap N$. It follows that $K N / K$ is a Lie group. Also, $G / K N$ is a Lie group since $G / N$ is a Lie group. Hence $G / K$ is a Lie group since $G / K N \cong(G / K) /(K N / K)$.

THEOREM 10. Let $G$ be a locally compact group which has a maximal compact normal subgroup $K$. Let $H$ be a closed normal subgroup of $G$ such that $G / H$ has a compact normal subgroup with Lie factor group. If $H$ satisfies one of the following conditions, then $G / K$ is a Lie group.

(I) $H$ is $\sigma$-compact and compact extensions of $H$ have maximal compact normal subgroups with Lie factor groups.

(II) $H$ is compactly generated nilpotent.

(III) $H$ is almost connected. 
Proof. We use Lemma 9 to prove this theorem. If $N / H$ is a compact normal subgroup of $G / H$ with Lie factor group, then $G / N$ is a Lie group and hence conditions (1) and (3) of Lemma 9 are satisfied for (I), (II), and (III). Condition (2) of Lemma 9 is satisfied trivially for (I); is satisfied for (II) using Theorem 8; and is satisfied for (III) since $N$ is almost connected.

The compactly generated condition on $H$ in (II) seems necessary. In [1, Example 2], $K=\{e\}, H=\sum H_{i}$, and $G / H$ is compact. The maximal compact normal subgroup of $H$ is the identity and $H$ is a Lie group, but $G / K$ is not a Lie group.

\section{REFERENCES}

1. R. W. Bagley and T. S. Wu, Maximal compact normal subgroups and pro-Lie groups, Proc. Amer. Math. Soc. 93 (1985), 373-376.

2. S. Grosser and M. Moskowitz, Compactness conditions in topological groups, J. Reine Angew. Math. 246 (1971), 1-40.

3. K. H. Hofmann, J. R. Liukkonen and M. W. Mislove, Compact extensions of compactly generated nilpotent groups are pro-Lie, Proc. Amer. Math. Soc. 84 (1982), 443-448.

4. J. R. Liukkonen, Dual spaces of groups with precompact conjugacy classes, Trans. Amer. Math. Soc. 180 (1973), 85-108.

5. D. Montgomery and L. Zippin, Topological transformation groups, Interscience, New York, 1955.

6. G. D. Mostow, Self-adjoint groups, Ann. of Math. 62 (1955), 44-55.

7. S. P. Wang, Compactness properties of topological groups. II, Duke Math. J. 39 (1972), 243-251.

8. T. S. Wu and Y. K. Yu, Compactness properties of topological groups, Michigan Math. J. 19 (1972), 299-313.

Department of Mathematics and Computer Science, University of Miami, CORAL GABLES, FLORIDA 33124 